\title{
Network-Based $H_{\infty}$ Filter Design for Linear System with Random Delays
}

\author{
Ruixia Yan, ${ }^{1}$ Zhong Wu, $^{1}$ and Jinliang Liu $^{2}$ \\ ${ }^{1}$ School of Management, Shanghai University of Engineering Science, Shanghai 201620, China \\ ${ }^{2}$ Department of Applied Mathematics, Nanjing University of Finance and Economics, Nanjing, Jiangsu 210046, China \\ Correspondence should be addressed to Jinliang Liu; liujinliang2009@163.com
}

Received 9 January 2013; Accepted 13 February 2013

Academic Editor: Yang Tang

Copyright (c) 2013 Ruixia Yan et al. This is an open access article distributed under the Creative Commons Attribution License, which permits unrestricted use, distribution, and reproduction in any medium, provided the original work is properly cited.

\begin{abstract}
This paper investigates the filtering problem for a class of network-based systems with random network-induced delays. The considered random delay between the sensor and the filter is assumed to be satisfying a certain stochastic characteristic. Considering the probability of the delay taking value in different intervals, a stochastic variable satisfying Bernoulli random binary distribution is introduced and a new system model is established by employing the information of the probability distribution. By using a properly constructed Lyapunov function, sufficient conditions for the existence of the $H_{\infty}$ filters are presented in terms of linear matrix inequalities, which are dependent on the occurrence probability of both the random communication delays. The filter parameter is then characterized by the solution to a set of LMIs. A simulation example is employed to show the effectiveness of the proposed method.
\end{abstract}

\section{Introduction}

With the wide application of networks in the complex dynamical processes such as advanced aircraft and manufacturing processes, the networked control systems (NCSs) have attracted great attention during the past few years, see [15]. However, they also present some challenging problems arising from the fact that data typically travel through the communication networks from sensors to the controller and from controller to the actuators. In particular, due to the finite bandwidth for data transmission over networks, random delay and packets loss are inevitable in networked systems where a common medium is used for data transfers. In many situations, they can even destabilize the system. Hence, the uncertainties in the measurement transmission have attracted increasing interest.

In recent years, lots of outstanding results have been published on sensor delays, packet dropouts, and missing measurements, see [6-8]. In [6], based on a Markov chain, the authors considered mixed uncertainties in the measurement transmission and developed a unified/combined model to accommodate random delay, packet dropouts and missing measurements. The authors in [7] invested a class of linear uncertain discrete-time stochastic systems with randomly varying sensor delay. The authors in [8] studied a delaydependent $H_{\infty}$ filtering for Markovian jump systems with time-varying delays, and criterion was derived for the $H_{\infty}$ performance analysis of the filtering-error systems, which could lead to much less conservative analysis results. The filtering problem for network-based systems with random delay and packets loss has attracted increasing attention during the past years, see [9-14]. For example, in [9], the filtering problem of network-based system with long timevarying delay was considered. The authors in [12] studied the robust filtering problem for a class of uncertain discrete time-delay systems with missing measurements. In [13], the authors designed $H_{\infty}$ filter for nonlinear systems with timedelay via a T-S fuzzy model approach.

However, to the best of our knowledge, the filtering problems for NCSs with random communication delays and missing measurements has not been fully investigated yet, which still remains as a challenging research issue in the literature. Moreover, the filtering problem for network-based systems with random delay and event triggered scheme 
has not been investigated in the existing literature, which motivates our present work.

In this paper, the filtering problem is addressed for communication delays and missing measurements. The delay is modeled as a Bernoulli process. By using the Lyapunov method and the LMI technique, a sufficient condition to guarantee the filtering error system exponentially meansquare stable is derived. A convex optimization problem is also formulated to design the optimal $H_{\infty}$ filter. A simulation example is used to demonstrate the effectiveness of the proposed design procedures.

Notation. $\mathbb{R}^{n}$ and $\mathbb{R}^{n \times m}$ denote the $n$-dimensional Eculidean space, and the set of $n \times m$ real matrices; the superscript " $T$ " stands for matrix transposition; $I$ is the identity matrix of appropriate dimension; $\|\cdot\|$ stands for the Euclidean vector norm or the induced matrix 2-norm as appropriate; the notation $X>0$ (resp., $X \geq 0$ ), for $X \in \mathbb{R}^{n \times n}$ means that the matrix $X$ is real symmetric positive definite (resp., positive semidefinite). When $x$ is a stochastic variable, $\mathbb{E}\{x\}$ stands for the expectation of $x$. For a matrix $B$ and two symmetric matrices $A$ and $C,\left[\begin{array}{ll}A & * \\ B & C\end{array}\right]$ denotes a symmetric matrix, where * denotes the entries implied by symmetry.

\section{System Description}

In this paper, we consider the following system:

$$
\begin{gathered}
x(k+1)=A x(k)+B w(k), \\
y(k)=C x(k), \\
z(k)=L x(k),
\end{gathered}
$$

where $x(k) \in \mathbb{R}^{n}, y(k) \in \mathbb{R}^{m}$, and $z(k) \in \mathbb{R}^{q}$ denote the state vector, measurement vector, and the signal vector to be estimated, respectively; $A, B, C$, and $L$ are parameter matrices with appropriate dimensions. We consider the following full order linear dynamic filter:

$$
\begin{gathered}
x_{f}(k+1)=A_{F} x_{f}(k)+B_{F} \hat{y}(k), \\
z_{f}(k)=C_{F} x_{f}(k)+D_{F} \hat{y}(k),
\end{gathered}
$$

where $x_{f}(k) \in \mathbb{R}^{n}$ is the filter state, $\hat{y}(k) \in \mathbb{R}^{m}$ is the filter input, and $z_{f}(k) \in \mathbb{R}^{n}$ is the estimated signal, and $A_{f}, B_{f}$, $C_{f}$, and $L_{f}$ are filter parameters to be determined.

We use $\tau(k)$ to denote the time-delay in the network between the sensor and the filter, and it is assumed that $\tau(k)$ takes values in $\left[\tau_{1}, \tau_{2}\right]$.

Assumption 1. $\tau(k)$ changes randomly and for a constant $\tau_{0} \in$ $\left[\tau_{0}, \tau_{2}\right]$, the probability of $\tau(k) \in\left[\tau_{1}, \tau_{0}\right]$ and $\tau(k) \in\left[\tau_{0}, \tau_{2}\right]$ can be known.

Define two sets as follows:

$$
\begin{aligned}
& \Omega_{1}=\left\{\tau(k) \in\left[\tau_{1}, \tau_{0}\right]\right\}, \\
& \Omega_{2}=\left\{\tau(k) \in\left[\tau_{0}, \tau_{2}\right]\right\} .
\end{aligned}
$$

Furthermore, two functions are defined as follows:

$$
\begin{gathered}
\tau_{1}(k)= \begin{cases}\tau(k), & \text { for } \tau(k) \in \Omega_{1} \\
0, & \text { for } \tau(k) \notin \Omega_{1}\end{cases} \\
\tau_{2}(k)= \begin{cases}\tau(k), & \text { for } \tau(k) \in \Omega_{2} \\
0, & \text { for } \tau(k) \notin \Omega_{2} .\end{cases}
\end{gathered}
$$

Obviously, $\Omega_{1} \cup \Omega_{2}=\mathbb{R}^{+}$and $\Omega_{1} \bigcap \Omega_{2}=\Phi$ (empty set).

From the definitions of $\Omega_{1}$ and $\Omega_{2}$, it can be seen that $\tau(k) \in \Omega_{1}$ means the event $\tau(k) \in\left[\tau_{1}, \tau_{0}\right]$ occurs and $\tau(k) \in$ $\Omega_{2}$ means the event $\tau(k) \in\left[\tau_{0}, \tau_{2}\right]$ occurs. Corresponding to $\tau(k)$ taking values in different intervals, a stochastic variable $\beta(k)$ is defined as

$$
\beta(k)= \begin{cases}1, & \tau(k) \in \Omega_{1} \\ 0, & \tau(k) \in \Omega_{2} .\end{cases}
$$

Assumption 2. $\beta(k)$ is a Bernoulli distributed sequence with

$$
\begin{gathered}
\operatorname{Prob}\{\beta(k)=1\}=\mathbb{E}\{\beta(k)\}=\beta_{0}, \\
\operatorname{Prob}\{\beta(k)=0\}=1-\mathbb{E}\{\beta(k)\}=1-\beta_{0},
\end{gathered}
$$

where $0 \leq \beta_{0} \leq 1$ is a constant.

Remark 3. The introduction of $\beta(k)$ is motivated by $[7,12$, 15 ], where the Bernoulli distributed sequence $\beta(k)$ is used to model the missing message of the systems. Different from [7, $12,15], \beta(k)$ is used in this paper to describe the time-varying delay taking values in different intervals.

Remark 4. From Assumption 2, it can be shown that $\mathbb{E}\{\beta(k)-$ $\left.\beta_{0}\right\}=0$ and $\mathbb{E}\left\{\left(\beta(k)-\beta_{0}\right)^{2}\right\}=\beta_{0}\left(1-\beta_{0}\right)$. Since $\operatorname{Prob}\{\tau(k) \epsilon$ $\left.\left[\tau_{1}, \tau_{0}\right]\right\}=\operatorname{Prob}\{\beta(k)=1\}$ and $\operatorname{Prob}\left\{\tau(k) \in\left[\tau_{0}, \infty\right]\right\}=$ $\operatorname{Prob}\{\beta(k)=0\}, \beta_{0}$ and $1-\beta_{0}$, respectively, denote the probability of $\tau(k)$ taking values in $\left[\tau_{1}, \tau_{0}\right]$ and $\left[\tau_{0}, \tau_{2}\right]$.

By using the new functions $\tau_{i}(k)(i=1,2)$ and $\beta(k)$, the filter input $\widehat{y}$ can be expressed as

$$
\begin{aligned}
\widehat{y}(k) & =y(k-\tau(k)) \\
& =\beta(k) C x\left(k-\tau_{1}(k)\right)+(1-\beta(k)) C x\left(k-\tau_{2}(k)\right) .
\end{aligned}
$$

Then, (4) can be rewritten as follows:

$$
\begin{aligned}
x_{f}(k+1)= & A_{F} x_{f}(k)+\beta(k) B_{F} C x\left(k-\tau_{1}(k)\right) \\
& +(1-\beta(k)) B_{F} C x\left(k-\tau_{2}(k)\right) \\
z_{f}(k)= & C_{F} x_{f}(k)+\beta(k) D_{F} C x\left(k-\tau_{1}(k)\right) \\
& +(1-\beta(k)) D_{F} C x\left(k-\tau_{2}(k)\right) .
\end{aligned}
$$


Define $\bar{x}(k)=\left[\begin{array}{c}x(k) \\ x_{f}(k)\end{array}\right]$ and $e(k)=z(k)-z_{f}(k)$, combining (1), (3), and (10), we can obtain the filtering-error system as follows:

$$
\begin{aligned}
\bar{x}(k+1)= & \bar{A} \bar{x}(k)+\beta(k) \bar{C} x\left(k-\tau_{1}(k)\right) \\
& +(1-\beta(k)) \bar{C} x\left(k-\tau_{2}(k)\right)+\bar{B} w(k) \\
e(k)= & \bar{L} \bar{x}(k)-\beta(k) D_{F} C x\left(k-\tau_{1}(k)\right) \\
& -(1-\beta(k)) D_{F} C x\left(k-\tau_{2}(k)\right),
\end{aligned}
$$

where $\bar{A}=\left[\begin{array}{cc}A & 0 \\ 0 & A_{f}\end{array}\right], \bar{C}=\left[\begin{array}{c}0 \\ B_{f} C\end{array}\right], \bar{B}=\left[\begin{array}{l}B \\ 0\end{array}\right]$, and $\bar{L}=\left[\begin{array}{ll}L & -C_{F}\end{array}\right]$.

Before giving the main result, we first introduce the following useful definitions and lemmas.

Definition 5. The system (1)-(3) are said to be exponentially stable in the mean-square sense (ESMSS), if there exist constants $\alpha>0, \lambda>0$, such that $t>0$

$$
\mathbb{E}\left\{\|x(t)\|^{2}\right\} \leq \alpha e^{-\lambda t} \sup _{-\tau_{M}<s<0}\left\{\|\phi(s)\|^{2}\right\} .
$$

Definition 6. System (11)-(12) are said to be asymptotically stable with an $H_{\infty}$ norm bound $\gamma$, if the following conditions hold:

(i) The filtering error system (11)-(12) with $w(t)=0$ is asymptotically stable.

(ii) Under the assumption of zero initial condition, the controlled output $e(t)$ satisfies $\|e(t)\|_{2} \leq \gamma\|w(t)\|_{2}$ for any nonzero $w(t) \in \mathscr{L}_{2}\left[t_{0}, \infty\right)$.

We recall the following lemmas that will be needed in the proof of our main results.

Lemma 7 (see [16]). For any vectors $x, y \in R^{n}$, and positive definite matrix $Q \in R^{n \times n}$, the following inequality holds:

$$
2 x^{T} y \leq x^{T} Q x+y^{T} Q^{-1} y .
$$

Lemma 8 (see [17]). For given matrix $Q>0$ and $\tau_{k}, x(k)$, $y(k)(k=1,2, \ldots)$, let $\delta(k)=x(k+1)-x(k)$, then the following four inequalities holds:

$$
\begin{aligned}
&-\tau_{k} \sum_{i=k-\tau_{k}}^{k-1} \delta^{T}(i) Q \delta(i) \\
& \quad \leq\left[\begin{array}{c}
x(k) \\
x\left(k-\tau_{k}\right)
\end{array}\right]^{T}\left[\begin{array}{cc}
-Q & Q \\
Q & -Q
\end{array}\right]\left[\begin{array}{c}
x(k) \\
x\left(k-\tau_{k}\right)
\end{array}\right] .
\end{aligned}
$$

Lemma 9 (see [18]). $\Xi_{11}, \Xi_{12}, \Xi_{21}, \Xi_{22}$, and $\Omega$ are constant matrices of appropriate dimensions, and $\tau_{i}(k)$ is function of $k$ and satisfies $\tau_{1} \leq \tau_{1}(k) \leq \tau_{0} \leq \tau_{2}(k) \leq \tau_{2}, i=1,2$, then

$$
\begin{aligned}
& {\left[\left(\tau_{1}(k)-\tau_{1}\right) \Xi_{11}+\left(\tau_{0}-\tau_{1}(k)\right) \Xi_{21}\right]} \\
& \quad+\left[\left(\tau_{2}(k)-\tau_{0}\right) \Xi_{12}+\left(\tau_{2}-\tau_{2}(k)\right) \Xi_{22}\right]+\Omega<0
\end{aligned}
$$

if and only if the following four inequalities hold:

$$
\begin{gathered}
\left(\tau_{0}-\tau_{1}\right) \Xi_{11}+\left(\tau_{2}-\tau_{0}\right) \Xi_{12}+\Omega<0 \\
\left(\tau_{0}-\tau_{1}\right) \Xi_{11}+\left(\tau_{2}-\tau_{0}\right) \Xi_{22}+\Omega<0 \\
\left(\tau_{0}-\tau_{1}\right) \Xi_{21}+\left(\tau_{2}-\tau_{0}\right) \Xi_{12}+\Omega<0 \\
\left(\tau_{0}-\tau_{1}\right) \Xi_{21}+\left(\tau_{2}-\tau_{01}\right) \Xi_{22}+\Omega<0 .
\end{gathered}
$$

Our purpose is to develop filters of the form (4) such that the filtering error system (11) and (12) are asymptotically stable in the mean-square sense with an $H_{\infty}$ disturbance attenuation level $\gamma$.

\section{Main Results}

The following theorem can be obtained for system (11) and (12).

Theorem 10. For some given constants $\tau_{1} \leq \tau_{0} \leq \tau_{2}$ and $\gamma$, the system (11) and (12) are ESMSS if there exist matrices $P>0$, $Q_{1}>0, Q_{2}>0, Q_{3}>0, R_{1}>0, R_{2}>0, R_{3}>0$ and matrices $N, M, T, S$ of appropriate dimensions, for $s=1,2,3,4$, such that

$$
\left[\begin{array}{ccc}
\Xi_{11}+\Gamma+\Gamma^{T} & * & * \\
\Xi_{21} & \Xi_{22} & * \\
\Xi_{31}(s) & 0 & \Xi_{33}
\end{array}\right]<0,
$$

where

$$
\begin{aligned}
& \Xi_{11}=\left[\begin{array}{ccccccc}
\Upsilon_{1} & * & * & * & * & * & * \\
R_{1} H & -Q_{1}-R_{1} & * & * & * & * & * \\
\beta_{0} \bar{C}^{T} P & 0 & 0 & * & * & * & * \\
0 & 0 & 0 & -Q_{3} & * & * & * \\
\left(1-\beta_{0}\right) \bar{C}^{T} P & 0 & 0 & 0 & 0 & * & * \\
0 & 0 & 0 & 0 & 0 & -Q_{2} & * \\
\bar{B}^{T} P & 0 & 0 & 0 & 0 & 0 & -\gamma^{2} I X
\end{array}\right]
\end{aligned}
$$

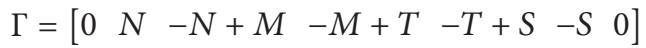




$$
\begin{aligned}
& \Xi_{21}=\left[\begin{array}{ccccccc}
\sqrt{\beta_{0}} P \bar{A} & 0 & \sqrt{\beta_{0}} P \bar{C} & 0 & 0 & 0 & \sqrt{\beta_{0}} P \bar{B} \\
\sqrt{1-\beta_{0}} P \bar{A} & 0 & 0 & 0 & \sqrt{1-\beta_{0}} P \bar{C} & 0 & \sqrt{1-\beta_{0}} P \bar{B} \\
\sqrt{\beta_{0}} \widetilde{R} H(\bar{A}-I) & 0 & \sqrt{\beta_{0}} \widetilde{R} H \bar{C} & 0 & 0 & 0 & \sqrt{\beta_{0}} \widetilde{R} H \bar{B} \\
\sqrt{1-\beta_{0}} \widetilde{R} H(\bar{A}-I) & 0 & 0 & 0 & \sqrt{1-\beta_{0}} \widetilde{R} H \bar{C} & 0 & \sqrt{1-\beta_{0}} \widetilde{R} H \bar{B} \\
\sqrt{\beta_{0} L} & 0 & -\sqrt{\beta_{0}} D_{F} C & 0 & 0 & 0 & 0 \\
\sqrt{1-\beta_{0} L} & 0 & 0 & 0 & -\sqrt{1-\beta_{0}} D_{F} C & 0 & 0
\end{array}\right] \\
& \Xi_{22}=\text { daig }\{-P,-P,-\widetilde{R},-\widetilde{R},-I,-I\} \\
& \Xi_{31}(1)=\left[\begin{array}{l}
\sqrt{\tau_{0}-\beta_{1}} N^{T} \\
\sqrt{\tau_{2}-\beta_{0}} T^{T}
\end{array}\right], \quad \Xi_{31}(2)=\left[\begin{array}{c}
\sqrt{\tau_{0}-\beta_{1}} N^{T} \\
\sqrt{\tau_{2}-\beta_{0}} S^{T}
\end{array}\right], \\
& \Xi_{31}(3)=\left[\begin{array}{c}
\sqrt{\tau_{0}-\beta_{1}} M^{T} \\
\sqrt{\tau_{2}-\beta_{0}} S^{T}
\end{array}\right], \quad \Xi_{31}(4)=\left[\begin{array}{c}
\sqrt{\tau_{0}-\beta_{1}} M^{T} \\
\sqrt{\tau_{2}-\beta_{0}} T^{T}
\end{array}\right] \\
& \Xi_{33}=\text { daig }\left\{-R_{2},-R_{3}\right\} \\
& \Upsilon_{1}=P \bar{A}+\bar{A}^{T} P+H^{T}\left(Q_{1}+Q_{2}+Q_{3}-R_{1}\right) H \\
& \widetilde{R}=\tau_{1}^{2} R_{1}+\left(\tau_{0}-\tau_{1}\right) R_{2}+\left(\tau_{2}-\tau_{0}\right) R_{3}, \quad H=\left[\begin{array}{ll}
I & 0
\end{array}\right] .
\end{aligned}
$$

Proof. Define

$$
\begin{aligned}
\delta(k)= & x(k+1)-x(k) \\
= & H[\bar{x}(k+1)-x(k)] \\
= & H\left[(\bar{A}-I) \bar{x}(k)+\beta(k) \bar{C} x\left(k-\tau_{1}(k)\right)\right. \\
& \left.\quad+(1-\beta(k)) \bar{C} x\left(k-\tau_{2}(k)\right)+\bar{B} w(k)\right] .
\end{aligned}
$$

Choose the following Lyapunov functional candidate as

$$
V(k)=V_{1}(k)+V_{2}(k)+V_{3}(k),
$$

where

$$
\begin{gathered}
V_{1}(k)=\bar{x}^{T}(k) P \bar{x}(k) \\
V_{2}(k)=\sum_{i=k-\tau_{1}}^{k-1} x^{T}(i) Q_{1} x(i)+\sum_{i=k-\tau_{2}}^{k-1} x^{T}(i) Q_{2} x(i) \\
+\sum_{i=k-\tau_{0}}^{k-1} x^{T}(i) Q_{3} x(i) \\
V_{3}(k)=\sum_{i=-\tau_{1}} \sum_{j=k+i}^{k-1} \delta^{T}(j) R_{1} \delta(j)+\sum_{i=-\tau_{0}}^{-\tau_{1}-1} \sum_{j=k+i}^{k-1} \delta^{T}(j) R_{2} \delta(j) \\
+\sum_{i=-\tau_{2}}^{-\tau_{0}-1} \sum_{j=k+i}^{k-1} \delta^{T}(j) R_{3} \delta(j) .
\end{gathered}
$$

Let $\Delta V_{i}(k)=V_{i}(k+1)-V_{i}(k), i=1,2,3$. Then along the system (11), we have

$$
\begin{aligned}
& \mathbb{E} \Delta V_{1}(k)=\mathbb{E}\left\{2 \bar{x}^{T}(k) P \bar{x}(k)+\bar{x}^{T}(k) P \bar{x}(k)\right\} \\
& =2 x^{T}(k) P\left[\bar{A} \bar{x}(k)+\beta_{0} \bar{C} x\left(k-\tau_{1}(k)\right)\right. \\
& \left.+\left(1-\beta_{0}\right) \bar{C} x\left(k-\tau_{2}(k)\right)+\bar{B} w(k)\right] \\
& +\beta_{0}\left[\bar{A} \bar{x}(k)+\bar{C} x\left(k-\tau_{1}(k)\right)+\bar{B} w(k)\right]^{T} \\
& \times P\left[\bar{A} \bar{x}(k)+\bar{C} x\left(k-\tau_{1}(k)\right)+\bar{B} w(k)\right] \\
& +\left(1-\beta_{0}\right)\left[\bar{A} \bar{x}(k)+\bar{C} x\left(k-\tau_{2}(k)\right)+\bar{B} w(k)\right]^{T} \\
& \times P\left[\bar{A} \bar{x}(k)+\bar{C} x\left(k-\tau_{2}(k)\right)+\bar{B} w(k)\right] \\
& \mathbb{E} \Delta V_{2}(k)=\bar{x}^{T}(k)\left[H^{T}\left(Q_{1}+Q_{2}+Q_{3}\right) H\right] \bar{x}(k) \\
& -x^{T}\left(k-\tau_{1}\right) Q_{1} x\left(k-\tau_{1}\right) \\
& -x^{T}\left(k-\tau_{2}\right) Q_{2} x\left(k-\tau_{2}\right) \\
& -x^{T}\left(k-\tau_{0}\right) Q_{3} x\left(k-\tau_{0}\right) \\
& \mathbb{E} \Delta V_{3}(k)=\mathbb{E}\left\{\delta^{T}(k) \widetilde{R} \delta(k)\right\}-\tau_{1} \sum_{i=k-\tau_{1}}^{k-1} \delta^{T}(i) R_{1} \delta(i) \\
& -\sum_{i=k-\tau_{0}}^{k-\tau_{1}-1} \delta^{T}(i) R_{2} \delta(i)-\sum_{i=k-\tau_{2}}^{k-\tau_{0}-1} \delta^{T}(i) R_{3} \delta(i)
\end{aligned}
$$

where $P>0, Q_{i}>0$ and $R_{i}>0, i=1,2,3$. 
Note that

$\mathbb{E}\left\{\delta^{T}(k) \widetilde{R} \delta(k)\right\}=\beta_{0} \Lambda_{1}^{T} H^{T} \widetilde{R} H \Lambda_{1}+\left(1-\beta_{0}\right) \Lambda_{2}^{T} H^{T} \widetilde{R} H \Lambda_{2}$,

where

$$
\begin{aligned}
& \Lambda_{1}=(\bar{A}-I) \bar{x}(k)+\bar{C} x\left(k-\tau_{1}(k)\right)+\bar{B} w(k), \\
& \Lambda_{2}=(\bar{A}-I) \bar{x}(k)+\bar{C} x\left(k-\tau_{2}(k)\right)+\bar{B} w(k) .
\end{aligned}
$$

By Lemma 8, we get

$$
\begin{aligned}
& -\tau_{1} \sum_{i=k-\tau_{1}}^{k-1} \delta^{T}(i) R_{1} \delta(i) \\
& \quad \leq\left[\begin{array}{c}
\bar{x}(k) \\
x\left(k-\tau_{1}\right)
\end{array}\right]^{T}\left[\begin{array}{cc}
-H^{T} R_{1} H & H^{T} R_{1} \\
R_{1} H & -R_{1}
\end{array}\right]\left[\begin{array}{c}
\bar{x}(k) \\
x\left(k-\tau_{1}\right)
\end{array}\right] .
\end{aligned}
$$

Employing the free-weighting matrices method [3], we have

$$
2 \xi^{T}(k) N\left[x\left(k-\tau_{1}\right)-x\left(k-\tau_{1}(k)\right)-\sum_{i=k-\tau_{1}(k)}^{k-\tau_{1}-1} \delta(i)\right]=0
$$

$$
\begin{aligned}
& 2 \xi^{T}(k) M\left[x\left(k-\tau_{1}(k)\right)-x\left(k-\tau_{0}\right)-\sum_{i=k-\tau_{0}}^{k-\tau_{1}(k)-1} \delta(i)\right]=0 \\
& 2 \xi^{T}(k) T\left[x\left(k-\tau_{0}\right)-x\left(k-\tau_{2}(k)\right)-\sum_{i=k-\tau_{2}(k)}^{k-\tau_{0}-1} \delta(i)\right]=0 \\
& 2 \xi^{T}(k) S\left[x\left(k-\tau_{2}(k)\right)-x\left(k-\tau_{2}\right)-\sum_{i=k-\tau_{2}}^{k-\tau_{2}(k)-1} \delta(i)\right]=0,
\end{aligned}
$$

where

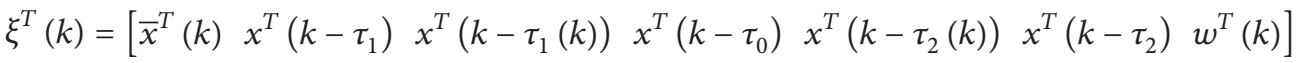

$$
\begin{aligned}
& N^{T}=\left[\begin{array}{llllllll}
N_{1}^{T} & N_{2}^{T} & N_{3}^{T} & N_{4}^{T} & N_{5}^{T} & N_{6}^{T} & N_{7}^{T}
\end{array}\right] \\
& M^{T}=\left[\begin{array}{lllllll}
M_{1}^{T} & M_{2}^{T} & M_{3}^{T} & M_{4}^{T} & M_{5}^{T} & M_{6}^{T} & M_{7}^{T}
\end{array}\right] \\
& T^{T}=\left[\begin{array}{lllllll}
T_{1}^{T} & T_{2}^{T} & T_{3}^{T} & T_{4}^{T} & T_{5}^{T} & T_{6}^{T} & T_{7}^{T}
\end{array}\right] \\
& S^{T}=\left[\begin{array}{lllllll}
S_{1}^{T} & S_{2}^{T} & S_{3}^{T} & S_{4}^{T} & S_{5}^{T} & S_{6}^{T} & S_{7}^{T}
\end{array}\right]
\end{aligned}
$$

By Lemma 7, there exist $R_{2}$ and $R_{3}$, such that

$$
\begin{aligned}
& -2 \xi^{T}(k) N \sum_{i=k-\tau_{1}(k)}^{k-\tau_{1}-1} \delta(i) \leq\left(\tau_{1}(k)-\tau_{1}\right) \xi^{T}(k) N R_{2}^{-1} N^{T} \xi(k) \\
& +\sum_{i=k-\tau_{1}(k)}^{k-\tau_{1}-1} \delta^{T}(i) R_{2} \delta(i) \\
& -2 \xi^{T}(k) M \sum_{i=k-\tau_{0}}^{k-\tau_{1}(k)-1} \delta(i) \leq\left(\tau_{0}-\tau_{1}(k)\right) \xi^{T}(k) M R_{2}^{-1} M^{T} \xi(k) \\
& +\sum_{i=k-\tau_{0}}^{k-\tau_{1}(k)-1} \delta^{T}(i) R_{2} \delta(i)
\end{aligned}
$$$$
-2 \xi^{T}(k) T \sum_{i=k-\tau_{2}(k)}^{k-\tau_{0}-1} \delta(i) \leq\left(\tau_{2}(k)-\tau_{0}\right) \xi^{T}(k) T R_{3}^{-1} T^{T} \xi(k)
$$$$
+\sum_{i=k-\tau_{2}(k)}^{k-\tau_{0}-1} \delta^{T}(i) R_{3} \delta(i)
$$

$$
\begin{aligned}
& -2 \xi^{T}(k) S \sum_{i=k-\tau_{2}}^{k-\tau_{2}(k)-1} \delta(i) \leq\left(\tau_{2}-\tau_{2}(k)\right) \xi^{T}(k) S R_{3}^{-1} S^{T} \xi(k) \\
& +\sum_{i=k-\tau_{2}}^{k-\tau_{2}(k)-1} \delta^{T}(i) R_{3} \delta(i) .
\end{aligned}
$$

By computing, we obtain

$$
\begin{aligned}
e^{T}(k) e(k)= & \beta_{0}\left(\bar{L} \bar{x}(k)-D_{F} C x\left(k-\tau_{1}(k)\right)\right)^{T} \\
& \times\left(\bar{L} \bar{x}(k)-D_{F} C x\left(k-\tau_{1}(k)\right)\right) \\
& +\left(1-\beta_{0}\right)\left(\bar{L} \bar{x}(k)-D_{F} C x\left(k-\tau_{2}(k)\right)\right)^{T} \\
& \times\left(\bar{L} \bar{x}(k)-D_{F} C x\left(k-\tau_{2}(k)\right)\right) .
\end{aligned}
$$

Combining (23)-(32), we have

$$
\begin{gathered}
\mathbb{E}\left\{\Delta V(k)-\gamma^{2} w^{T}(k) w(k)+e^{T}(k) e(k)\right\} \\
\leq 2 x^{T}(k) P\left[\bar{A} \bar{x}(k)+\beta_{0} \bar{C} x\left(k-\tau_{1}(k)\right)\right. \\
\left.+\left(1-\beta_{0}\right) \bar{C} x\left(k-\tau_{2}(k)\right)+\bar{B} w(k)\right] \\
+\beta_{0}\left[\bar{A} \bar{x}(k)+\bar{C} x\left(k-\tau_{1}(k)\right)+\bar{B} w(k)\right]^{T}
\end{gathered}
$$




$$
\begin{aligned}
& \times P\left[\bar{A} \bar{x}(k)+\bar{C} x\left(k-\tau_{1}(k)\right)+\bar{B} w(k)\right] \\
& +\left(1-\beta_{0}\right)\left[\bar{A} \bar{x}(k)+\bar{C} x\left(k-\tau_{2}(k)\right)+\bar{B} w(k)\right]^{T} \\
& \times P\left[\bar{A} \bar{x}(k)+\bar{C} x\left(k-\tau_{2}(k)\right)+\bar{B} w(k)\right] \\
& +\bar{x}^{T}(k)\left[H^{T}\left(Q_{1}+Q_{2}+Q_{3}\right) H\right] \bar{x}(k) \\
& -x^{T}\left(k-\tau_{1}\right) Q_{1} x\left(k-\tau_{1}\right)-x^{T}\left(k-\tau_{2}\right) Q_{2} x\left(k-\tau_{2}\right) \\
& -x^{T}\left(k-\tau_{0}\right) Q_{3} x\left(k-\tau_{0}\right)+\beta_{0} \Lambda_{1}^{T} H^{T} \widetilde{R} H \Lambda_{1} \\
& +\left(1-\beta_{0}\right) \Lambda_{2}^{T} H^{T} \widetilde{R} H \Lambda_{2} \\
& +\left[\begin{array}{c}
\bar{x}(k) \\
x\left(k-\tau_{1}\right)
\end{array}\right]^{T}\left[\begin{array}{cc}
-H^{T} R_{1} H & H^{T} R_{1} \\
R_{1} H & -R_{1}
\end{array}\right]\left[\begin{array}{c}
\bar{x}(k) \\
x\left(k-\tau_{1}\right)
\end{array}\right] \\
& +2 \xi^{T}(k) N\left[x\left(k-\tau_{1}\right)-x\left(k-\tau_{1}(k)\right)\right] \\
& +2 \xi^{T}(k) M\left[x\left(k-\tau_{1}(k)\right)-x\left(k-\tau_{0}\right)\right] \\
& +2 \xi^{T}(k) T\left[x\left(k-\tau_{0}\right)-x\left(k-\tau_{2}(k)\right)\right] \\
& +2 \xi^{T}(k) S\left[x\left(k-\tau_{2}(k)\right)-x\left(k-\tau_{2}\right)\right] \\
& +\left(\tau_{1}(k)-\tau_{1}\right) \xi^{T}(k) N R_{2}^{-1} N^{T} \xi(k) \\
& +\left(\tau_{0}-\tau_{1}(k)\right) \xi^{T}(k) M R_{2}^{-1} M^{T} \xi(k) \\
& +\left(\tau_{2}(k)-\tau_{0}\right) \xi^{T}(k) T R_{3}^{-1} T^{T} \xi(k) \\
& +\left(\tau_{2}-\tau_{2}(k)\right) \xi^{T}(k) S R_{3}^{-1} S^{T} \xi(k)-\gamma^{2} w^{T}(k) w(k)
\end{aligned}
$$

$$
\begin{aligned}
& +\beta_{0}\left(\bar{L} \bar{x}(k)-D_{F} C x\left(k-\tau_{1}(k)\right)\right)^{T} \\
& \times\left(\bar{L} \bar{x}(k)-D_{F} C x\left(k-\tau_{1}(k)\right)\right) \\
& +\left(1-\beta_{0}\right)\left(\bar{L} \bar{x}(k)-D_{F} C x\left(k-\tau_{2}(k)\right)\right)^{T} \\
& \times\left(\bar{L} \bar{x}(k)-D_{F} C x\left(k-\tau_{2}(k)\right)\right) .
\end{aligned}
$$

Then, by using well-known Schur complement and Lemma 9, from (33), one can easily see that (18) with $s=$ $1,2,3,4$ can lead $\mathbb{E}\left\{\Delta V(k)-\gamma^{2} w^{T}(k) w(k)+e^{T}(k) e(k)\right\}<0$. The remaining part of the proof is similar to those in [19] and so omitted here for simplicity. The proof is complete.

In the following, based on Theorem 10, we will design the filter parameters $A_{F}, B_{F}, C_{F}, D_{F}$, in (4).

Theorem 11. For some given constants $\tau_{1} \leq \tau_{0} \leq \tau_{2}$ and $\gamma$, the system (11) and (12) are ESMSS if there exist matrices $P>0$, $P_{3}>0, Q_{1}>0, Q_{2}>0, Q_{3}>0, R_{1}>0, R_{2}>0, R_{3}>0$ and matrices $\bar{A}_{f}, \bar{B}_{f}, \bar{C}_{f}, \bar{D}_{f}, \bar{N}_{10}, \bar{N}_{11}, \bar{M}_{10}, \bar{M}_{11}, \bar{T}_{10}, \bar{T}_{11}, \bar{S}_{10}$, $\bar{S}_{11}, N_{i}, M_{i}, T_{i}, S_{i},(i=1,2, \ldots, 7)$ of appropriate dimensions such that the following LMIs hold:

$$
\begin{gathered}
{\left[\begin{array}{ccc}
\bar{\Xi}_{11} & * & * \\
\bar{\Xi}_{21} & \bar{\Xi}_{22} & * \\
\bar{\Xi}_{31}(s) & 0 & \Xi_{33}
\end{array}\right]<0} \\
P_{1}-\bar{P}_{3}>0
\end{gathered}
$$

$$
\begin{aligned}
& \bar{\Xi}_{11}=\left[\begin{array}{cccccccc}
\bar{\Gamma}_{1} & * & * & * & * & * & * & * \\
\bar{P}_{3} A+\bar{A}_{F}^{T} & \bar{A}_{F}+\bar{A}_{F}^{T} & * & * & * & * & * & * \\
R_{1}+N_{10}^{T} & N_{11}^{T} & \bar{\Gamma}_{2} & * & * & * & * & * \\
\bar{\Gamma}_{3} & \bar{\Gamma}_{4} & N_{3}-N_{2}^{T}+M_{2}^{T} & \bar{\Gamma}_{5} & * & * & * & * \\
-M_{10}^{T}+T_{10}^{T} & -M_{11}^{T}+T_{11}^{T} & N_{4}-M_{2}^{T}+T_{2}^{T} & \bar{\Gamma}_{6} & \bar{\Gamma}_{7} & * & * & * \\
\bar{\Gamma}_{8} & \bar{\Gamma}_{9} & N_{5}-T_{2}^{T}+S_{2}^{T} & \bar{\Gamma}_{10} & \bar{\Gamma}_{11} & \bar{\Gamma}_{12} & * & * \\
-S_{10}^{T} & -S_{11}^{T} & N_{6}-S_{2}^{T} & -N_{6}+M_{6}-S_{3}^{T} & \bar{\Gamma}_{13} & \bar{\Gamma}_{14} & \bar{\Gamma}_{15} & * \\
B^{T} P_{1} & B^{T} \bar{P}_{3}^{T} & N_{7} & -N_{7}+M_{7} & -M_{7}+T_{7} & -T_{7}+S_{7} & -S_{7} & -\gamma^{2} I
\end{array}\right] \\
& \bar{\Xi}_{21}=\left[\begin{array}{cccccccc}
\sqrt{\beta_{0}} P_{1} A & \sqrt{\beta_{0}} \bar{A}_{F} & 0 & \sqrt{\beta_{0}} \bar{B}_{F} C & 0 & 0 & 0 & \sqrt{\beta_{0}} P_{1} B \\
\sqrt{\beta_{0}} \bar{P}_{3} A & \sqrt{\beta_{0}} \bar{A}_{F} & 0 & \sqrt{\beta_{0}} \bar{B}_{F} C & 0 & 0 & 0 & \sqrt{\beta_{0}} \bar{P}_{3} B \\
\sqrt{1-\beta_{0}} P_{1} A & \sqrt{\beta_{0}} \bar{A}_{F} & 0 & 0 & 0 & \sqrt{1-\beta_{0}} \bar{B}_{F} C & 0 & \sqrt{1-\beta_{0}} P_{1} B \\
\sqrt{1-\beta_{0}} \bar{P}_{3} A & \sqrt{\beta_{0}} \bar{A}_{F} & 0 & 0 & 0 & \sqrt{1-\beta_{0}} \bar{B}_{F} C & 0 & \sqrt{1-\beta_{0}} \bar{P}_{3} B \\
\sqrt{\beta_{0}} \widetilde{R}(A-I) & 0 & 0 & 0 & 0 & 0 & 0 & \sqrt{\beta_{0}} \widetilde{R} B \\
\sqrt{1-\beta_{0}} \widetilde{R}(A-I) & 0 & 0 & 0 & 0 & 0 & 0 & \sqrt{\beta_{0}} \widetilde{R} B \\
\sqrt{\beta_{0}} L & -\sqrt{\beta_{0}} \bar{C}_{F} & 0 & -\sqrt{\beta_{0}} \bar{D}_{F} C & 0 & 0 & 0 & 0 \\
\sqrt{1-\beta_{0}} L & -\sqrt{1-\beta_{0}} \bar{C}_{F} & 0 & 0 & 0 & -\sqrt{1-\beta_{0}} \bar{D}_{F} C & 0 & 0
\end{array}\right]
\end{aligned}
$$




$$
\begin{aligned}
& \bar{\Xi}_{31}(1)=\left[\begin{array}{c}
\sqrt{\tau_{0}-\beta_{1}} N^{T} \\
\sqrt{\tau_{2}-\beta_{0}} \bar{T}^{T}
\end{array}\right], \quad \bar{\Xi}_{31}(2)=\left[\begin{array}{c}
\sqrt{\tau_{0}-\beta_{1} N}{ }^{T} \\
\sqrt{\tau_{2}-\beta_{0}} S^{T}
\end{array}\right], \\
& \bar{\Xi}_{31}(3)=\left[\begin{array}{c}
\sqrt{\tau_{0}-\beta_{1}} \bar{M}^{T} \\
\sqrt{\tau_{2}-\beta_{0}} \bar{S}^{T}
\end{array}\right], \quad \bar{\Xi}_{31}(4)=\left[\begin{array}{c}
\sqrt{\tau_{0}-\beta_{1}} \bar{M}^{T} \\
\sqrt{\tau_{2}-\beta_{0}} \bar{T}^{T}
\end{array}\right] \\
& \bar{\Xi}_{33}=\operatorname{diag}\{-\widetilde{P},-\widetilde{P},-\widetilde{R},-\widetilde{R},-I,-I\} \\
& \bar{\Gamma}_{1}=P_{1} A+A^{T} P_{1}+Q_{1}+Q_{2}+Q_{3}-R_{1}, \\
& \bar{\Gamma}_{2}=-Q_{1}-R_{1}+N_{2}+N_{2}^{T} \\
& \bar{\Gamma}_{3}=\beta_{0} C^{T} \bar{B}_{F}^{T}-N_{10}^{T}+M_{10}^{T} \text {, } \\
& \bar{\Gamma}_{4}=\beta_{0} C^{T} \bar{B}_{F}^{T}-N_{11}^{T}+M_{11}^{T} \\
& \bar{\Gamma}_{5}=-N_{3}-N_{3}^{T}+M_{3}+M_{3}^{T} \text {, } \\
& \bar{\Gamma}_{6}=-N_{4}+M_{4}-M_{3}^{T}+T_{3}^{T} \\
& \bar{\Gamma}_{7}=-Q_{3}-M_{4}-M_{4}^{T}+T_{4}+T_{4}^{T} \text {, } \\
& \bar{\Gamma}_{8}=\left(1-\beta_{0}\right) C^{T} \bar{B}_{F}^{T}-T_{10}^{T}+S_{10}^{T} \\
& \bar{\Gamma}_{9}=\left(1-\beta_{0}\right) C^{T} \bar{B}_{F}^{T}-T_{11}^{T}+S_{11}^{T} \text {, } \\
& \bar{\Gamma}_{10}=-N_{5}+M_{5}-T_{3}^{T}+S_{3}^{T} \\
& \bar{\Gamma}_{11}=-M_{5}+T_{5}-T_{4}^{T}+S_{4}^{T} \text {, } \\
& \bar{\Gamma}_{12}=-T_{5}-T_{5}^{T}+S_{5}+S_{5}^{T} \\
& \bar{\Gamma}_{13}=-M_{6}+T_{6}-S_{4}^{T}, \quad \bar{\Gamma}_{14}=-T_{6}+S_{6}-S_{5}^{T}, \\
& \bar{\Gamma}_{15}=-Q_{2}-S_{6}-S_{6}^{T} \\
& \bar{N}^{T}=\left[\begin{array}{lllllllll}
N_{10}^{T} & N_{11}^{T} & N_{2}^{T} & N_{3}^{T} & N_{4}^{T} & N_{5}^{T} & N_{6}^{T} & N_{7}^{T}
\end{array}\right] \\
& \bar{M}^{T}=\left[\begin{array}{lllllllll}
M_{10}^{T} & M_{11}^{T} & M_{2}^{T} & M_{3}^{T} & M_{4}^{T} & M_{5}^{T} & M_{6}^{T} & M_{7}^{T}
\end{array}\right] \\
& \bar{T}^{T}=\left[\begin{array}{llllllll}
T_{10}^{T} & T_{11}^{T} & T_{2}^{T} & T_{3}^{T} & T_{4}^{T} & T_{5}^{T} & T_{6}^{T} & T_{7}^{T}
\end{array}\right] \\
& \bar{S}^{T}=\left[\begin{array}{llllllll}
S_{10}^{T} & S_{11}^{T} & S_{2}^{T} & S_{3}^{T} & S_{4}^{T} & S_{5}^{T} & S_{6}^{T} & S_{7}^{T}
\end{array}\right] \\
& \widetilde{P}=\left[\begin{array}{cc}
-P_{1} & * \\
-\bar{P}_{3} & -\bar{P}_{3}
\end{array}\right] \text {. }
\end{aligned}
$$

Moreover, if the above conditions are feasible, the parameter matrices of the filter are given by

$$
\begin{gathered}
A_{F}=\bar{A}_{F} \bar{P}_{3}^{-1}, \\
B_{F}=\bar{B}_{F}, \\
C_{F}=\bar{C}_{F} \bar{P}_{3}^{-1}, \\
D_{F}=\bar{D}_{F} .
\end{gathered}
$$

Proof. Since $\bar{P}_{3}>0$, there exist $P_{2}$ and $P_{3}>0$ satisfying $\bar{P}_{3}=$ $P_{2}^{T} P_{3}^{-1} P_{2}$.

Define

$$
\begin{gathered}
P=\left[\begin{array}{ll}
P_{1} & P_{2}^{T} \\
P_{2} & P_{3}
\end{array}\right], \quad J=\left[\begin{array}{cc}
I & 0 \\
0 & P_{2}^{T} P_{3}^{-1}
\end{array}\right], \\
\Pi=\operatorname{dig}\{J, \underbrace{I, \ldots, I}_{6}, J, J, \underbrace{I, \ldots, I}_{6}\} .
\end{gathered}
$$

By schur complement, it is easy to see that $P>0$ is equivalent to $P_{1}-\bar{P}_{3}=P_{1}-P_{2}^{T} P_{3}^{-1} P_{2}>0$.

Pre- and postmultiplying (18) by $\Pi$ and its transpose, respectively, and let

$$
\begin{gathered}
\bar{A}_{F}=\widehat{A}_{F} \bar{P}_{3}^{-1}, \quad \widehat{A}_{F}=P_{2}^{T} A_{F} P_{2}^{-T} \\
\bar{B}_{F}=P_{2}^{T} B_{F} \\
\bar{C}_{F}=\widehat{C}_{F} \bar{P}_{3}^{-1}, \quad \widehat{C}_{F}=C_{F} P_{2}^{-T} \\
\bar{D}_{F}=D_{F} \\
N_{1}^{T} J^{T}=\left[\begin{array}{ll}
\bar{N}_{10}^{T} & \bar{N}_{11}^{T}
\end{array}\right] \\
M_{1}^{T} J^{T}=\left[\begin{array}{ll}
\bar{M}_{10}^{T} & \bar{M}_{11}^{T}
\end{array}\right] \\
T_{1}^{T} J^{T}=\left[\begin{array}{ll}
\bar{T}_{10}^{T} & \bar{T}_{11}^{T}
\end{array}\right] \\
S_{1}^{T} J^{T}=\left[\begin{array}{ll}
\bar{S}_{10}^{T} & \bar{S}_{11}^{T}
\end{array}\right] .
\end{gathered}
$$

Then, (18) for $s=1,2,3,4$ are equivalent to (34) for $s=$ $1,2,3,4$, respectively.

Next, we will show that, if (34) and (35) are solvable for $\bar{A}_{F}, \bar{B}_{F}, \bar{C}_{F}, \bar{D}_{F}$ and $\bar{P}_{3}$, then the parameter matrices of the filter (4) can be chosen as in (38).

Replacing $\left(\bar{A}_{F}, \bar{B}_{F}, \bar{C}_{F}, \bar{D}_{F}\right)$ by $\left(P_{2}^{-T} \widehat{A}_{F} P_{2}^{T}, P_{2}^{-T} \bar{B}_{F}, \widehat{C}_{F} P_{2}^{T}\right.$, $\bar{D}_{F}$ ) in (34) and then pre- and post-multiplying them with $\Pi$ and $\Pi^{T}$, we can also obtain (34). Obviously, $\left(P_{2}^{-T} \widehat{A}_{F} P_{2}^{T}\right.$, $\left.P_{2}^{-T} \bar{B}_{F}, \widehat{C}_{F} P_{2}^{T}, \bar{D}_{F}\right)$ can be chosen as the filter parameters. That is, the following filter

$$
\begin{gathered}
\tilde{x}(k+1)=P_{2}^{-T} \widehat{A}_{F} P_{2}^{T} \tilde{x}(k)+P_{2}^{-T} \bar{B}_{F} \tilde{y}(k) \\
\tilde{r}(k)=\widehat{C}_{F} P_{2}^{T} \tilde{x}(k)+\bar{D}_{F} \tilde{y}(k)
\end{gathered}
$$

can guarantee that the filter system (11) and (12) are asymptotically stable with the $H_{\infty}$ performance bound $\gamma$. Defining $\widehat{x}(k)=P_{2}^{T} \widetilde{x}(k),(41)$ becomes

$$
\begin{gathered}
\widehat{x}(k+1)=\widehat{A}_{F} \widehat{x}(k)+\bar{B}_{F} \tilde{y}(k), \\
r(k)=\widehat{C}_{F} \widehat{x}(k)+\bar{D}_{F} \tilde{y}(k) .
\end{gathered}
$$

Then, from (40) and (42) we can obtain (38). This completes the proof. 


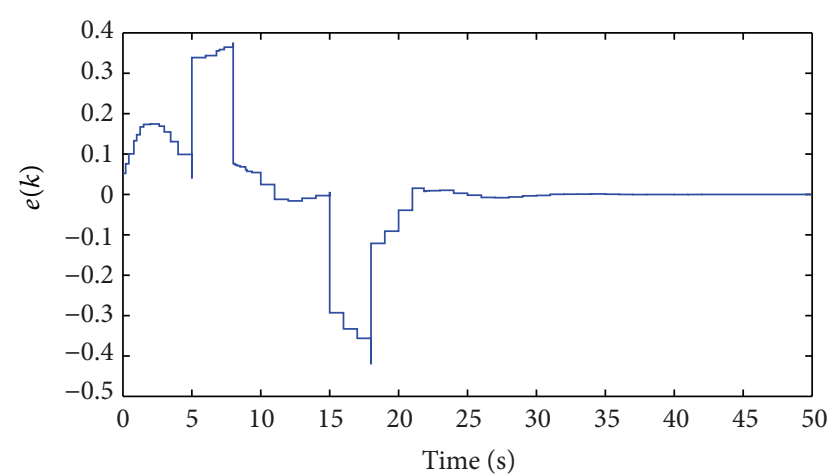

FIGURE 1: Estimated signals error $e(k)=z(k)-z_{f}(k)$.

\section{Simulation Examples}

Consider the system (1)-(3) with parameter matrices

$$
\begin{aligned}
& A=\left[\begin{array}{ll}
0.2 & 0.4 \\
0.4 & 0.2
\end{array}\right], \quad B=\left[\begin{array}{c}
-1 \\
1
\end{array}\right], \\
& C=\left[\begin{array}{ll}
1 & -1
\end{array}\right], \quad L=\left[\begin{array}{ll}
-1 & 1
\end{array}\right] .
\end{aligned}
$$

When $1 \leq \tau(k) \leq 4, \tau_{0}=2, \beta_{0}=7$, we can obtain the parameter matrices of the filter as follows:

$$
\begin{aligned}
& A=\left[\begin{array}{ll}
-0.4127 & 0.4231 \\
-1.2374 & 0.6783
\end{array}\right], \quad B_{F}=\left[\begin{array}{c}
-0.0129 \\
0.0237
\end{array}\right], \\
& C_{F}=\left[\begin{array}{ll}
-0.6778 & -0.2837
\end{array}\right], \quad D_{F}=-0.3427 .
\end{aligned}
$$

To illustrate the performance of the designed filter, choose the disturbance function as follows:

$$
w(k)= \begin{cases}-1, & 5 s<t<8 s \\ 1, & 15 s<t<18 s \\ 0, & \text { otherwise. }\end{cases}
$$

Figure 1 shows the error-estimation signal of $e(k)$, and Figure 2 shows the state of filtering-error system with the initial values $x^{T}(0)=\left[\begin{array}{ll}0.5 & -0.5\end{array}\right]$.

\section{Conclusion}

This paper has investigated the problem of filtering design for linear time-delay system. The probability distribution of the delay taking values in some intervals is assumed to be known a priori. Corresponding to the probability of the delay taking value in different intervals, a stochastic variable satisfying Bernoulli random binary distribution has been introduced and a new modeling method is presented to describe the overall filtering error system. Sufficient conditions are derived to guarantee the stochastic stability and a $H_{\infty}$ performance level for the filtering error system. An optimal filter design problem is also provided by optimizing the filtering performances. Simulations are conducted to evaluate and demonstrate the performance of the proposed approach.

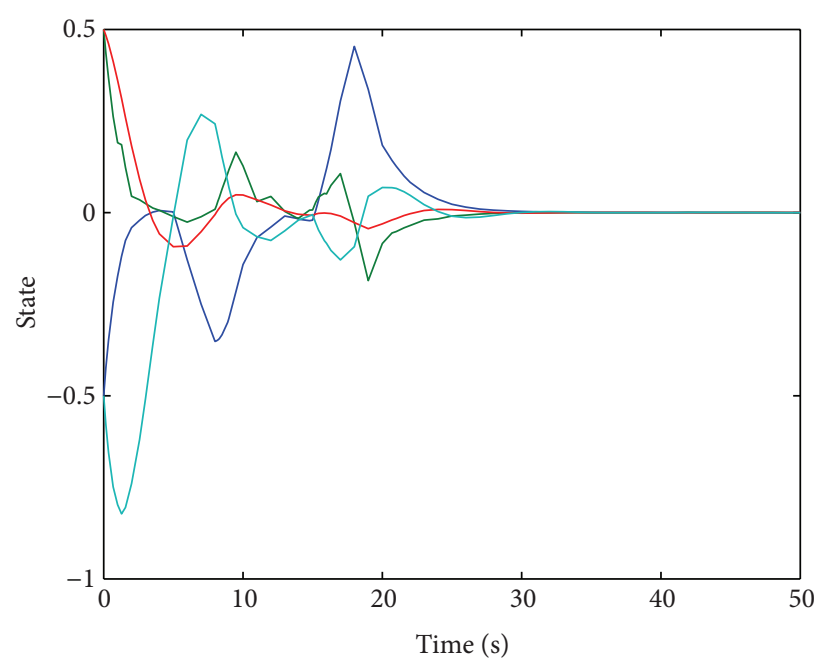

FIGURE 2: The state curves of filtering error system.

\section{Acknowledgments}

This work is partly supported by the National Natural Science Foundation of China (no. 11226240), the Natural Science Foundation of Jiangsu Province of China (no. BK2012469), the Natural Science Foundation of the Jiangsu Higher Education Institutions of China (no. 12 KJD120001), the Young Teacher Supporting Foundation of Shanghai (no. ZZGJD12036), and a Project Funded by the Priority Academic Program Development of Jiangsu Higher Education Institutions (PAPD).

\section{References}

[1] J. Liang, Z. Wang, and X. Liu, "State estimation for coupled uncertain stochastic networks with missing measurements and time-varying delays: the discrete-time case," IEEE Transactions on Neural Networks, vol. 20, no. 5, pp. 781-793, 2009.

[2] J. Liu, D. Yue, Z. Gu, and E. Tian, " $H_{\infty}$ filtering for systems with time-varying delay satisfying a certain stochastic characteristic," IET Signal Processing, vol. 5, no. 8, pp. 757-766, 2011.

[3] D. Yue, Q.-L. Han, and J. Lam, "Network-based robust $H_{\infty}$ control of systems with uncertainty," Automatica, vol. 41, no. 6, pp. 999-1007, 2005.

[4] J. Xiong and J. Lam, "Stabilization of linear systems over networks with bounded packet loss," Automatica, vol. 43, no. 1, pp. 80-87, 2007.

[5] C. Peng and Y.-C. Tian, "Delay-dependent robust stability criteria for uncertain systems with interval time-varying delay," Journal of Computational and Applied Mathematics, vol. 214, no. 2, pp. 480-494, 2008.

[6] Y. Tang, H. Gao, W. Zou, and J. Kurths, "Distributed synchronization in networks of agent systems with nonlinearities and random switchings," IEEE Transactions on Cybernetics, vol. 43, no. 1, pp. 358-370, 2013.

[7] Y. Tang, J. A. Fang, M. Xia, and D. Yu, "Delay-distributiondependent stability of stochastic discrete-time neural networks with randomly mixed time-varying delays," Neurocomputing, vol. 72, no. 16-18, pp. 3830-3838, 2009. 
[8] W. Zhang, Y. Tang, J. A. Fang, and X. Wu, "Stochastic stability of genetic regulatory networks with a finite set delay characterization," ChaosInterdisciplinary Journal of Nonlinear Science, vol. 22, no. 2, Article ID 023106, 2012.

[9] W. Zhang, J. A. Fang, and Y. Tang, "New robust stability analysis for genetic regulatory networks with random discrete delays and distributed delays," Neurocomputing, vol. 74, no. 14-15, pp. 2344-2360, 2011.

[10] M. Moayedi, Y. C. Soh, and Y. K. Foo, "Optimal kalman filtering with random sensor delays, packet dropouts and missing measurements," in Proceedings of the American Control Conference (ACC '09), pp. 3405-3410, IEEE, June 2009.

[11] Z. Wang, D. W. C. Ho, and X. Liu, "Robust filtering under randomly varying sensor delay with variance constraints," IEEE Transactions on Circuits and Systems II, vol. 51, no. 6, pp. 320326, 2004.

[12] J. Liu, B. Yao, and Z. Gu, "Delay-dependent $H_{\infty}$ filtering for Markovian jump time-delay systems: a piecewise analysis method," Circuits, Systems, and Signal Processing, vol. 30, no. 6, pp. 1253-1273, 2011.

[13] H. Song, L. Yu, and W. Zhang, " $H_{\infty}$ filtering of network-based systems with random delay," Signal Processing, vol. 89, no. 4, pp. 615-622, 2009.

[14] L. Schenato, "Optimal estimation in networked control systems subject to random delay and packet loss," in Proceedings of the 45th IEEE Conference on Decision and Control (CDC '06), pp. 5615-5620, IEEE, December 2006.

[15] M. Moayedi, Y. K. Foo, and Y. C. Soh, "Adaptive Kalman filtering in networked systems with random sensor delays, multiple packet dropouts and missing measurements," IEEE Transactions on Signal Processing, vol. 58, no. 3, pp. 1577-1588, 2010.

[16] Z. Wang, F. Yang, D. Ho, and X. Liu, "Robust H/sub/spl infin//filtering for stochastic time-delay systems with missing measurements," IEEE Transactions on Signal Processing, vol. 54, no. 7, pp. 2579-2587, 2006.

[17] J. Liu, S. Hu, H. Han, and R. Yan, " $H_{\infty}$ filter design for nonlinear systems with time-delay through T-S fuzzy model approach," in Proceedings of the Chinese Control and Decision Conference (CCDC '10), pp. 3606-3611, IEEE, May 2010.

[18] H. Gao, J. Lam, and C. Wang, "Robust energy-to-peak filter design for stochastic time-delay systems," Systems \& Control Letters, vol. 55, no. 2, pp. 101-111, 2006.

[19] F. Yang, Z. Wang, Y. S. Hung, and M. Gani, " $H_{\infty}$ control for networked systems with random communication delays," IEEE Transactions on Automatic Control, vol. 51, no. 3, pp. 511-518, 2006. 


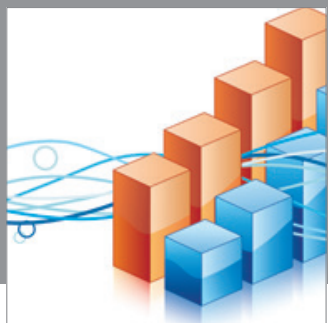

Advances in

Operations Research

mansans

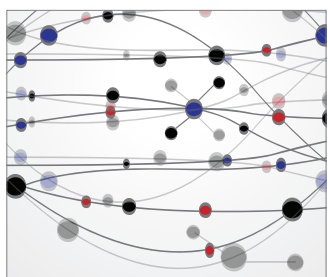

The Scientific World Journal
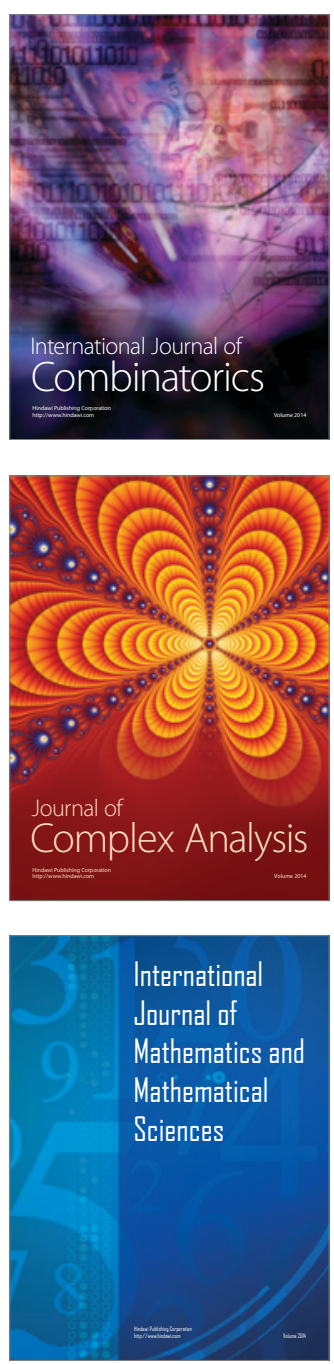
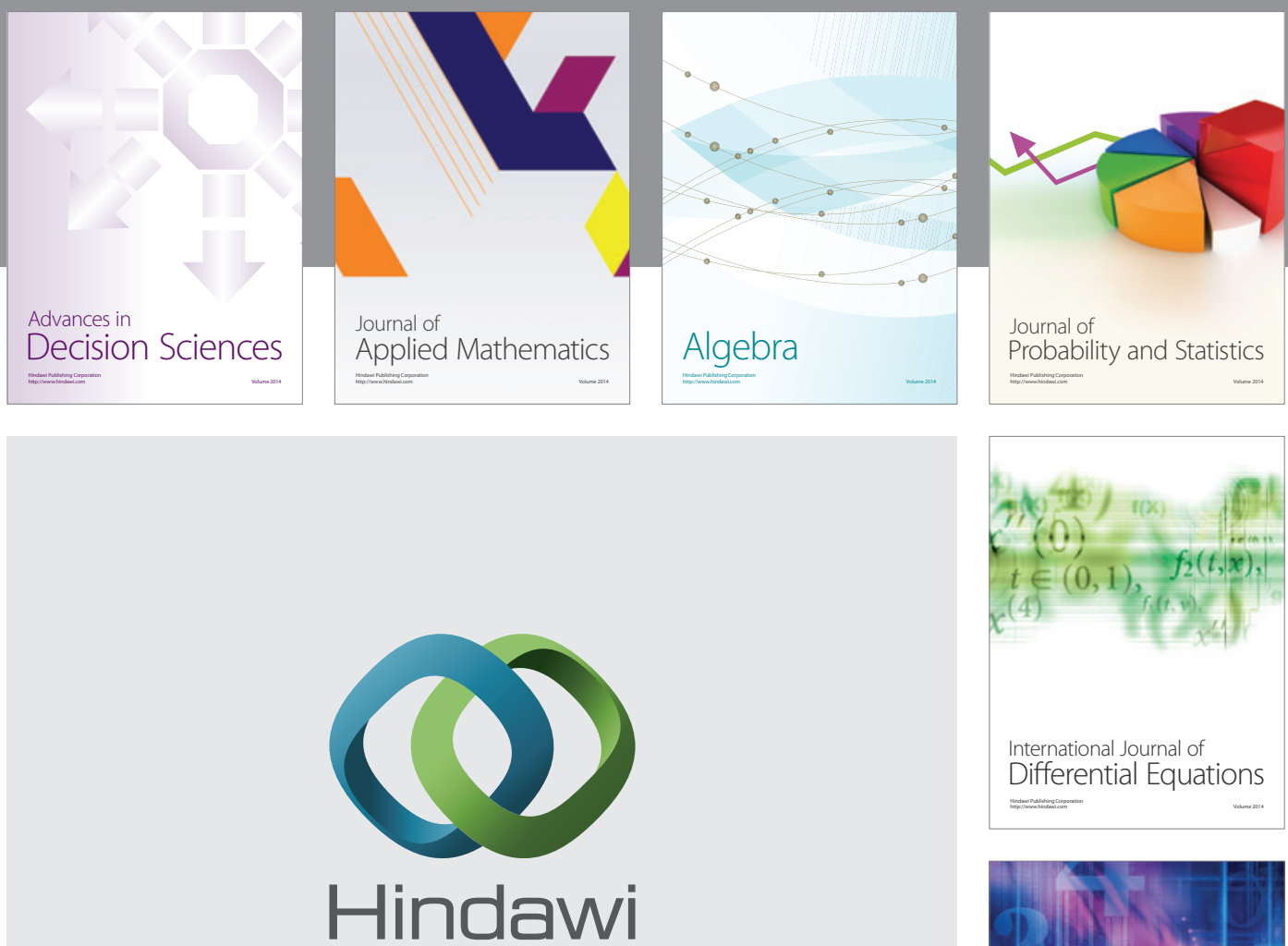

Submit your manuscripts at http://www.hindawi.com
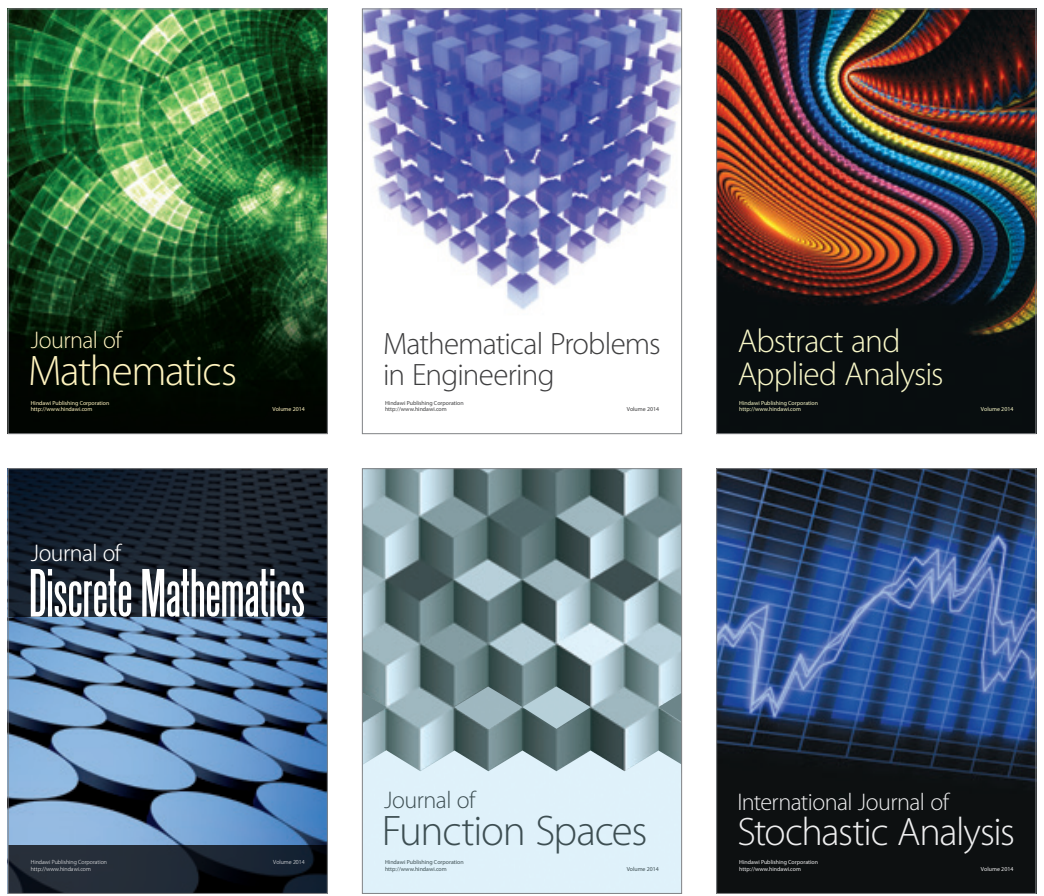

Journal of

Function Spaces

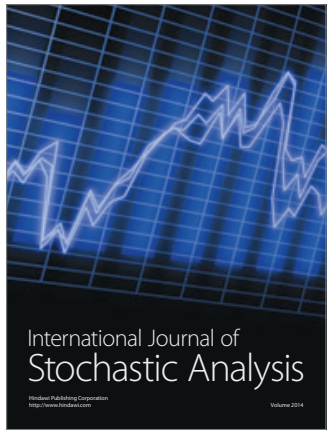

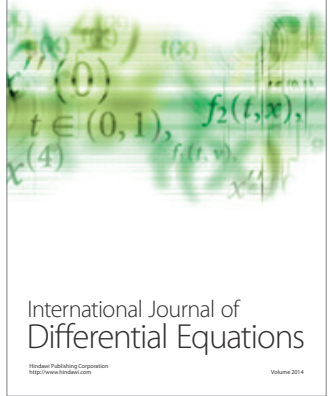
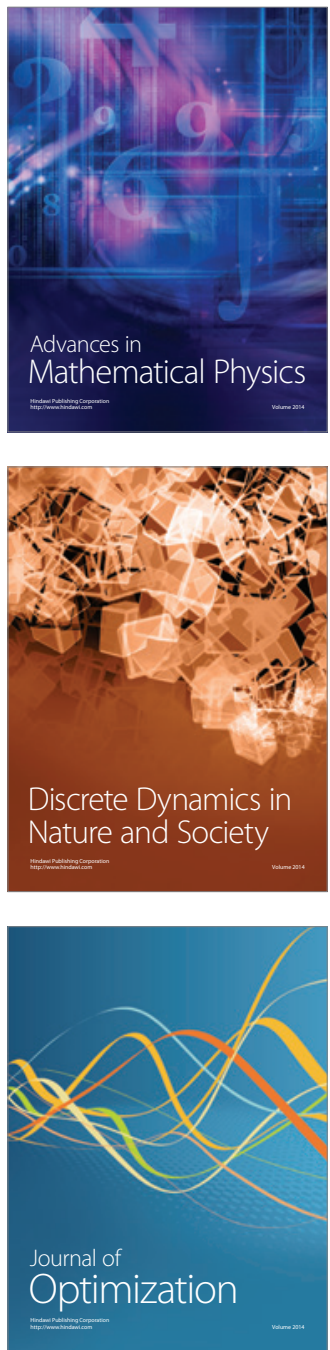